\title{
Human Telomerase Reverse Transcriptase (hTERT) Expression and Telomerase Activity in Acute and Chronic Lymphocytic Leukemia Patients
}

\author{
Dalia G. AMIN, Shahira K. ANIS, Sarah YOUNAN, Rasha adel Al GAMAL \\ Cairo University, Faculty of Medicine, Department of Clinical and Chemical Pathology, Cairo, EGYPT
}

\begin{abstract}
Telomerase activity is crucial for pathogenesis and progression of some hematological malignancies. Telomerase is composed of human telomerase reverse transcriptase (hTERT) and RNA component (hTR). Our aim was to determine hTERT expression and Telomerase activity in acute and chronic lymphocytic leukemia patients (ALL, CLL) and to study their correlation with clinical, hematological and available prognostic parameters. This case control study was conducted on 43 patients (24 B-ALL, 19 B-CLL), and 24 age and sex matched controls. Real-time quantitative polymerase chain reaction and PCR-based telomeric repeats amplification protocol were used for determination of hTERT expression and Telomerase activity, respectively. hTERT was expressed in 20/24 (83.3 \%) ALL patients, 12/19 (63.15\%) CLL patients with no significant difference in the incidence or the median expression of hTERT among both groups. ALL had a significantly higher telomerase activity compared to both CLL and controls, while the latter 2 groups showed no significant difference. Telomerase activity wasn't correlated with hTERT expression. hTERT expression was positively correlated with BM blast $\%$ in ALL patients and with the \% of CD38 ( $p=0.001)$ and ZAP $70 \%(p=0.001)$ in CLL patients. Telomerase activity was correlated positively with the absolute lymphocytic counts in CLL $(p=0.021)$ and negatively with the patient's age of both groups $(p=0.003)$. Developing new targeted therapy directed against telomerase and/or hTERT could ultimately be of special benefit in patients with high telomerase activity and/or hTERT expression among ALL and CLL patients.
\end{abstract}

Keywords: Telomerase activity, hTERT expression, B-ALL/CLL, qRT-PCR; TRAP

\section{INTRODUCTION}

Telomeric protective sequences which are located at the ends of each chromosome, shorten after each cellular replication. When telomere shortens to a critical point, DNA damage pathways are turned on resulting in cellular apoptosis. Telomere erosion and replicative apoptosis is a tumor-suppressive machinery. ${ }^{1}$

Telomerase enzyme which is responsible for telomeric ends expansion neutralizes the previous mechanism. $^{2}$ The two components of Telomerase module are; human telomerase reverse tran- scriptase (hTERT) and RNA template named human telomerase RNA component (hTR). HTERT expression level are strongly associated with telomerase activity. ${ }^{3}$

The majority of malignant tumors endure high telomerase activity, empowering the cells to escape apoptosis and divide infinitely. ${ }^{4}$ Telomerase activity appears as a fundamental contributor in the pathogenesis and progression of some hematological malignancies whereas in other hematologic neoplasm telomerase activity is not the initiator of disease, meanwhile it is related to progressive disease, worse prognosis, or chemotherapy resistance. ${ }^{5}$ 
B-cell chronic lymphocytic leukemia (B-CLL), which is one of the most prevalent adult leukemias, uniquely manifests with extremely diverse clinical course, whereas a category of patients deteriorate and die within few months from diagnosis, others live for many years with minimal or no treatment and may die from a cause other than CLL. Detailed prognostic profile of B-CLL patients and identification of novel prognostic markers is crucial for individually tailored therapeutic approaches. ${ }^{6}$

Acute lymphoblastic leukaemia (ALL) is the most prevalent malignancy in childhood representing $80 \%$ of childhood leukaemia. Scarce studies have studied telomerase activity or hTERT in paediatric leukaemic malignancies. ${ }^{7,8}$ Although prognosis of ALL in childhood was enhanced with new evolved chemotherapeutics, still a category of ALL patients with failure of remission induction or early relapse represent a poor prognostic challenge. It is postulated that new prognostic markers and novel therapeutic targets might contribute to improved therapeutic policies.

The objective of this study was to determine hTERT expression and Telomerase activity in BALL and B-CLL patients and to study the correlation of hTERT expression and Telomerase activity with different clinical, hematological and available prognostic disease parameters.

\section{PATIENTS AND METHODS}

This study was conducted on 43 patients, 24 with B-acute lymphoblastic leukemia (B-ALL) and 19 patients with B- chronic lymphocytic leukemia (BCLL), as well as 24 age and sex matched normal controls who attended the Out Patients Clinic of General Surgery for elective repair of hernia. These patients attended Kasr El Aini Hospital-Cairo University, to be diagnosed in the Clinical Pathology Department. Informed consent was obtained from each participant or his/her guardian before being enrolled in this study. The study was approved by the ethics committee of Cairo University. Detailed patients' characteristics are shown in Table 1.

\section{RNA isolation and cDNA preparation}

Total RNA was purified using the RNeasy Mini Kit (Qiagen GmbH, Germany) according to the manufacturer's protocol.
The integrity and the relative amount of RNA were evaluated through agarose gel electrophoresis and spectrophotometric measurements, respectively. RNA was retro-transcribed into cDNA using SuperscriptTM First-Strand Synthesis Systems for RTQ-PCR (Life Technologies) according to the manufacturer's instructions. Synthesized cDNA was stored at $-80^{\circ} \mathrm{C}$ till used.

\section{Determination of hTERT expression by real- time quantitative polymerase chain reaction (qRT-PCR)}

QRT-PCR was performed for hTERT and the endogenous control (GAPDH) on Applied Biosystems 7300 thermocycler (Qiagen, CA, USA) using specific primers and fluorogenic probes as described before. ${ }^{9}$ In brief, the $20 \mu \mathrm{L}$ amplification reactions contained $7 \mu \mathrm{L}$ cDNA, $10 \mu \mathrm{L}$ of the Universal Taqman 2X PCR mastermix (Applied Biosystems) and $1.0 \mu \mathrm{L}$ of each of the specific primers and probe. The thermal cycling conditions were set as follows; $2 \mathrm{~min}$ at $50^{\circ} \mathrm{C}, 10 \mathrm{~min}$ at $95^{\circ} \mathrm{C}$, followed by 40 cycles of $95^{\circ} \mathrm{C}$ for $15 \mathrm{sec}$, and $60^{\circ} \mathrm{C}$ for 1 minute. All reactions were performed in duplicate and the average cycle threshold (CT) was obtained. PCR controls without reverse transcriptase was routinely used as negative control. Results were provided as normalized hTERT expression, using the $\Delta \Delta \mathrm{Ct}$ method (the amount of target, normalized to an endogenous reference (GAPDH) and relative to the reference), target amount $=2-\Delta \Delta \mathrm{Ct}$ where $\Delta \Delta \mathrm{Ct}=[\mathrm{Ct}$ (hTERT target $)-\mathrm{Ct}(\mathrm{GAPDH}$ target $)-$ $\mathrm{Ct}$ (hTERT reference) - Ct (GAPDH reference)]9.

\section{Determination of telomerase activity by PCR- based telomeric repeats amplification protocol (TRAP)}

Peripheral blood mononuclear cells were tested for the presence of telomerase activity using telomerase PCR-ELISA (Roche Diagnostic GmbH) as reported previously. ${ }^{10}$ In short, each TRAP reaction contained $25 \mu \mathrm{l}$ reaction mixture (Tris-buffer, containing telomerase substrate, primers, nucleotides, and Taq polymerase), $2 \mu \mathrm{l}$ cell extract (corresponding to $2 \times 10^{3}$ ) and sterile water to make 50 $\mu 1$. The reaction mixture was incubated at $25^{\circ} \mathrm{C}$ for 


\begin{tabular}{|c|c|c|c|}
\hline Variable & ALL (24) & CLL (19) & Total \\
\hline \multicolumn{4}{|l|}{ Sex, Number (\%) } \\
\hline Male & 17 (58.6) & $12(41.4)$ & $29(67.4)$ \\
\hline Female & $7(50.0)$ & $7(50.0)$ & $14(32.6)$ \\
\hline \multicolumn{4}{|l|}{ Age, years } \\
\hline Median, Range & $24.0(13-60)$ & $60(40-79)$ & $31.0(13-79)$ \\
\hline \multicolumn{4}{|l|}{ Splenomegaly, Number (\%) } \\
\hline Yes & $10(55.6)$ & $8(44.4)$ & $18(41.9)$ \\
\hline No & $14(56.0)$ & $11(44.0)$ & $25(58.1)$ \\
\hline \multicolumn{4}{|l|}{ Hepatomegaly, Number (\%) } \\
\hline Yes & $3(75.0)$ & $1(25.0)$ & $4(9.3)$ \\
\hline No & $21(53.8)$ & $18(46.2)$ & $39(90.7)$ \\
\hline \multicolumn{4}{|l|}{ Lymphadenopathy, Number (\%) } \\
\hline Yes & $6(50.0)$ & $6(50.0)$ & $12(27.9)$ \\
\hline No & $18(58.1)$ & $13(41.9)$ & $31(72.1)$ \\
\hline Platelet X 109/L (Median,Range) & $129(18-292)$ & $123(10-1116)$ & $129(10-1116)$ \\
\hline \multicolumn{4}{|l|}{ Hemoglobin, g/dl } \\
\hline Mean \pm S. D. & $10.5 \pm 2.4$ & $9.7 \pm 2.7$ & $10.2 \pm 2.5$ \\
\hline TLC, x103/ul (Median, Range). & $7.0(0.7-817)$ & $48.7(9.3-626)$ & $15.3(0.7-817)$ \\
\hline Newly diagnosed & $13(54.2 \%)$ & $9(100 \%)$ & - \\
\hline Relapsed & 5 (20.8\%) & 0 & - \\
\hline Remission & $6(25 \%)$ & 0 & - \\
\hline PB blast \% (Median, Range) & $24.0(0-100)$ & - & - \\
\hline BM blast \% (Median, Range) & $86.0(0-100)$ & - & - \\
\hline Lymph\% (Median, Range) & - & $83.0(57.0-98)$ & - \\
\hline ALC x 1000 (Median, Range) & - & $29.4(5.3-330.2)$ & - \\
\hline Zap70 (Median, Range) & - & $40.0(4-80)$ & - \\
\hline CD38 (Median, Range) & - & $40.0(2-80)$ & - \\
\hline \multicolumn{4}{|l|}{ Quantitative hTERT } \\
\hline Median, Range & $2.2(0-61)$ & $2.6(0-48.1)$ & $2.6(0-61)$ \\
\hline \multicolumn{4}{|l|}{ Qualitative hTERT } \\
\hline Yes & $20(62.5)$ & $12(37.5)$ & $32(74.4)$ \\
\hline No & $4(36.4)$ & 7 (63.6) & $11(25.6)$ \\
\hline \multicolumn{4}{|l|}{ Telomerase activity } \\
\hline Median, Range & $39.5(0-348)$ & $19.5(0-1132)$ & $29(0-1132)$ \\
\hline
\end{tabular}

30 minutes and heated at $94^{\circ} \mathrm{C}$ for 5 minutes. Then PCR amplification for 30 cycles was performed. Each cycle consisted of denaturation at $94^{\circ} \mathrm{C}$ for 30 seconds, annealing at $50^{\circ} \mathrm{C}$ for 30 seconds, and polymerization at $72^{\circ} \mathrm{C}$ for 90 seconds, followed by extension at $72^{\circ} \mathrm{C}$ for 10 minutes. After PCR, $5 \mu \mathrm{l}$ of the PCR product was incubated with $20 \mu \mathrm{l}$ of denaturation reagent at room temperature for 10 minutes, vortexed briefly with $100 \mu 1$ hybridization buffer. One hundred $\mu \mathrm{l}$ of the mixture was transferred to each well of the precoated microplate, then incubated at $37^{\circ} \mathrm{C}$ on a shaker for 2 hours, washed 3 times and $100 \mu \mathrm{l}$ Anti-Dig- POD were added. After washing substrate solution was added and incubated at room temperature for 20 minutes. Using an ELISA reader, the absorbance values of the samples were measured at $450 \mathrm{~nm}$ within 30 minutes after $100 \mu \mathrm{l}$ of stop reagent was added. The mean absorbance of the negative controls was subtracted from those of the samples. Samples are regarded as telomerase- positive if the difference in absorbance $(\Delta \mathrm{A})$ is higher than 0.2. Negative samples were repeated. 
International Journal of Hematology and Oncology

Table 2. Comparison of different parameters in association with the hTERT expression pattern among the studied CLL patients

\begin{tabular}{|c|c|c|c|c|c|c|c|}
\hline \multirow{2}{*}{$\begin{array}{l}\text { CLL group } \\
\text { variants }\end{array}$} & \multicolumn{3}{|c|}{ Negative hTERT expression (7) } & \multicolumn{3}{|c|}{ Positive hTERT expression (12) } & \multirow[t]{2}{*}{$\mathbf{p}$} \\
\hline & Median & Minimum & Maximum & Median & Minimum & Maximum & \\
\hline Age, years & 60.0 & 50.0 & 70.0 & 58.0 & 40.0 & 79.0 & 0.482 \\
\hline $\mathrm{Hb} \mathrm{g} / \mathrm{dl}$ & 8.5 & 4.0 & 12.3 & 10.6 & 4.8 & 12.9 & 0.494 \\
\hline TLC $\times 1000$ & 49.5 & 16.0 & 626.0 & 40.2 & 9.3 & 355.0 & 0.616 \\
\hline Plat $\times 1000$ & 47.0 & 10.0 & 298.0 & 136.5 & 10.0 & 1116.0 & 0.385 \\
\hline Lymph\% & 76.5 & 60.0 & 98.0 & 85.0 & 57.0 & 93.0 & 0.340 \\
\hline ALC $\times 1000$ & 35.5 & 11.2 & 212.7 & 32.0 & 5.3 & 330.2 & 0.616 \\
\hline Zap70\% & 9.0 & 4.0 & 53.0 & 50.0 & 5.0 & 80.0 & 0.964 \\
\hline CD38\% & 10.5 & 2.0 & 68.0 & 59.0 & 2.0 & 80.0 & $0.020 *$ \\
\hline Telo A & 14.5 & 0.0 & 1132.0 & 21.0 & 5.8 & 130.0 & 0.098 \\
\hline
\end{tabular}

ALC= Absolute lymphocytic count, Telo A= Telomerase Activity, ${ }^{*}=\mathrm{p}<0.05$ statistically significant

\section{RESULTS}

hTERT was found to be expressed in 20 out of the $24(83.3 \%)$ ALL patients and 12 out of 19 (63.15\%) CLL patients with no significant difference in the incidence of expression among the ALL and CLL groups $(\mathrm{p}=0.722)$. The median expression of hTERT was 2.2 (range 0.0-61) in the ALL group, and 2.6 (range 0.0-48.1) in the CLL group with no significant difference $(\mathrm{p}=0.132)$. Control group did not show any expression of hTERT.

ALL patients showed a significantly higher median telomerase activity (39.5, range 0.0-348) compared to both CLL patients (19.5, range 0.0-1132) and controls $(13$, range $1.2-64),(p<0.001)$, while the latter 2 groups showed no significant difference regarding telomerase activity.

Among CLL patients, telomerase activity values weren't correlated with hTERT expression values $(p=0.923, r=0.024)$, while for ALL patients this was just on the cutoff ( $\mathrm{p}=0.05, \mathrm{r}=0.40$ ).

Comparing between CLL patients with positive hTERT expression versus those with negative expression regarding different clinical and laboratory variants, showed a significantly higher percent of CD38 in hTERT positive CLL group $(\mathrm{p}=0.02)$. No other significant difference could be elicited regarding (patient's age, hemoglobin percent, platelets count, total leucocytic count, lymphocyte $\%$, absolute lymphocytic counts or ZAP $70 \%$ ) between the 2 groups, despite the median Telomer- ase activity was higher among the hTERT positive group, however this couldn't reach a statistical significance $(\mathrm{p}=0.098)$ may be due to small sample size (Table 2).

The previous comparative study couldn't be applied for the ALL patients group as the number of ALL patients with negative hTERT expression was too small for statistical analysis.

Comparing different categories of ALL or CLL patients regarding the level of hTERT expression as well as the level of telomerase activity, didn't show any significant difference, this was elicited for the following categories (males/females, patients with organomegaly/patients with no organomegaly, patients with lymphadenopathy/ patients with no lymphadenopathy) (Table 3).

Concerning ALL patients; comparison between newly diagnosed, relapsed and those in remission as regards expression of hTERT and telomerase activity, showed no statistically significant difference $(\mathrm{p}=0.079,0.64)$ respectively, despite the median hTERT expression was evidently lower among the remission group, however this couldn't reach a statistical significance $(\mathrm{p}=0.079)$ (Table 3$)$.

hTERT expression was positively correlated with blast $\%$ in bone marrow (BM) of ALL patients ( $\mathrm{p}=$ $0.044)$, with the \% of CD38 ( $\mathrm{p}=0.001)$ and ZAP $70 \%(\mathrm{p}=0.001)$ in peripheral blood $(\mathrm{PB})$ of CLL patients, otherwise hTERT expression was not correlated with any other variable. Telomerase activ- 


\begin{tabular}{|c|c|c|c|c|c|c|c|c|c|c|}
\hline & \multirow[t]{2}{*}{ No } & \multicolumn{3}{|c|}{ hTERT expression } & & \multirow[t]{2}{*}{ p } & \multicolumn{3}{|c|}{ Telomerase Activity } & \multirow[t]{2}{*}{ p } \\
\hline & & Med & Min & Max & & & Med & Min & $\operatorname{Max}$ & \\
\hline \multirow[t]{2}{*}{ Sex } & Female & 14 & 3.0 & 0.1 & 61.0 & 0.264 & 32.5 & 0.0 & 153.0 & 0.678 \\
\hline & Male & 29 & 1.3 & 0.0 & 52.7 & & 29.0 & 0.0 & 1132.0 & \\
\hline \multirow[t]{2}{*}{ Hepatomegaly } & Y & 4 & 3.4 & 0.0 & 42.0 & 0.702 & 25.3 & 0.0 & 58.0 & 0.732 \\
\hline & $\mathrm{N}$ & 39 & 1.8 & 0.0 & 61.0 & & 29.0 & 0.0 & 1132.0 & \\
\hline \multirow[t]{2}{*}{ Splenomegaly } & Y & 18 & 3.0 & 0.0 & 61.0 & 0.204 & 23.5 & 0.0 & 153.0 & 0.398 \\
\hline & $\mathrm{N}$ & 25 & 1.0 & 0.0 & 50.2 & & 30.0 & 0.0 & 1132.0 & \\
\hline \multirow[t]{2}{*}{ Lymphadenopathy } & Y & 12 & 3.0 & 0.0 & 52.7 & 0.243 & 26.0 & 0.0 & 348.0 & 0.698 \\
\hline & $\mathrm{N}$ & 31 & 1.2 & 0.0 & 61.0 & & 29.0 & 0.0 & 1132.0 & \\
\hline \multirow[t]{3}{*}{ ALL group } & $\begin{array}{l}\text { Newly } \\
\text { diagnos }\end{array}$ & $d^{13}$ & 3.0 & 0.0 & 61.0 & 0.079 & 39.0 & 0.0 & 153.0 & 0.638 \\
\hline & \multicolumn{2}{|c|}{ Relapsed 5} & 3.0 & 0.0 & 50.2 & & 48.0 & 25.0 & 348.0 & \\
\hline & \multicolumn{2}{|c|}{ Remission } & 6 & 0.5 & 0.0 & 2.6 & & 35.5 & 12.2 & 48.0 \\
\hline
\end{tabular}

ity was positively correlated with the absolute lymphocytic counts among CLL group $(p=0.021)$ and negatively correlated with the patient's age of both leukemia groups $(p=0.003)$, otherwise it was not correlated with any other variable in both ALL and CLL patients, in addition no correlation was elicited between Telomerase activity and \% of CD38 or ZAP 70 among CLL patients) (Table 4).

\section{DISCUSSION}

Telomerase regulation is essential component in cellular aging and cancer. hTERT is crucial controlling subunit of telomerase in various cells. In this study we analyzed both Telomerase activity and hTERT expression in context with clinical, hematological as well as prognostic parameters among two groups of ALL and CLL patients.

The current study encountered hTERT expression in $83.3 \%$ of ALL patients and $63.15 \%$ of CLL patients, and none of the controls, with no difference in the incidence of expression among the two leukemia groups. In agreement, Tchirkov et al reported nearly the same incidence as ours (59\%) for the expression of hTERT gene among $90 \mathrm{~B}-\mathrm{CLL}$ patients. ${ }^{9}$ Meanwhile Terrin et al, reported a higher incidence $(74.6 \%)$ of hTERT expression in $134 \mathrm{~B}$ CLL patients. ${ }^{11}$ Also, a recent study by Abdelrahman et al, reported that hTERT mRNA was found to be expressed in all studied BCLL patients and none of the normal controls. ${ }^{12}$ These variant results may be referred to different amplified hTERT transcript as it has been established that hTERT has alternate splicing sites. ${ }^{13}$

As for ALL, considering the scarce studies that has analyzed hTERT expression in context with the ALL phenotype, Kleideiter et al. reported a nearby incidence of expression as ours $(90 \%, 18 / 20)$ among T-ALL patients. ${ }^{14}$ On the other hand Nowak et $\mathrm{al}^{15}$, reported the expression of hTERT in all the ALL pediatric patients in their study, and stated that majority of healthy individuals did not show any expression of telomerase components.

Our data showed no significant difference in telomerase activity between CLL patients and controls, while ALL patients had a significantly higher telomerase activity compared to both groups. All our reviewed studies have established that telomerase activity was elevated in ALL patients compared to healthy individuals. ${ }^{15-17}$ As for CLL patients, in support to our findings, Trentin et al reported that unlike most cancer cells, telomerase activity is not 
Table 4. Correlative study between different parameters of ALL/CLL patients in association with the level of hTERT expression as well as the level of telomerase activity

\begin{tabular}{|c|c|c|c|c|c|}
\hline & & \multicolumn{2}{|c|}{ q-hTERT expression } & \multicolumn{2}{|c|}{ Telomerase Activity } \\
\hline & & $\mathbf{r}$ & $\mathbf{p}$ & $\mathbf{r}$ & $\mathbf{p}$ \\
\hline \multirow[t]{4}{*}{ ALL, CLL groups } & Age, years & -0.149 & 0.340 & -0.442 & 0.003 \\
\hline & $\mathrm{Hb} \mathrm{g} / \mathrm{dl}$ & 0.150 & 0.341 & -0.160 & 0.311 \\
\hline & TLC X 1000 & 0.017 & 0.916 & -0.026 & 0.871 \\
\hline & Plat X 1000 & -0.109 & 0.492 & -0.215 & 0.172 \\
\hline \multirow[t]{2}{*}{ ALL group } & Blast \% PB & 0.374 & 0.079 & 0.255 & 0.240 \\
\hline & Blast \%BM & 0.423 & 0.044 & 0.246 & 0.259 \\
\hline \multirow[t]{4}{*}{ CLL group } & Lymph \% & 0.107 & 0.662 & 0.276 & 0.253 \\
\hline & ALC $\times 1000$ & -0.018 & 0.943 & 0.526 & 0.021 \\
\hline & Zap70 & 0.739 & 0.001 & 0.126 & 0.629 \\
\hline & CD38 & 0.712 & 0.001 & -0.174 & 0.503 \\
\hline
\end{tabular}

up regulated in CLL patients. ${ }^{18}$ Also it is demonstrated that telomerase activity is not vital for the origination of B-CLL, but it is essential for its maintenance. ${ }^{19}$

This study found no correlation between hTERT expression and telomerase activity in ALL or CLL patients. In accordance, Nowak et al, opposed any correlation between the degree of hTERT amplification and telomerase expression or activity among ALL patients. ${ }^{15}$ In contrast, it is reported that levels of hTERT mRNA significantly correlated with telomerase activity in ALL ${ }^{14}$ and CLL. ${ }^{20}$ To our explanation these discordant results between expression of hTERT and telomerase activity could be explained by transcriptional control of hTERT. As hTERT is alternatively spliced in heterogenous sites, these variant transcripts might create controlling machinery for telomerase activity, to be noted that spliced variants lacking $\mathrm{a}$ and $\mathrm{b}$ regions which carry the reverse transcriptase domains, can't encode a functional protein. ${ }^{13}$ It is worth mentioning that differential expression of hTERT splice variants is encountered in ALL at diagnosis as well as in remission. ${ }^{16}$

We encountered a significantly higher percent of CD38 in hTERT positive expression CLL group, with no significant difference regarding age, total leucocyte count, lymphocyte \%, absolute lymphocytic counts or ZAP 70 between hTERT positive and negative expression groups. In confirmation to our findings, hTERT expression values were signif- icantly higher in the CD38 positive CLL samples, meanwhile the same study reported that hTERT levels were higher in ZAP-70 positive cases than in the ZAP-70 negative cases despite being statistically insignificant. ${ }^{11}$

The present work didn't encounter any significant difference either in expression of hTERT or in telomerase activity among ALL patients groups (newly diagnosed, relapsed and remission).To our conclusion, the evidently lower hTERT expression in ALL patients who were in remission couldn't reach a statistical significance owing to the small number of the remission group (6 patients). Nowak et al, reported that telomerase activity in ALL patients in remission was at the level equivalent to that of normal individuals, and they explained the severe reduction of telomerase activity in remission by the elimination of blasts cells by chemotherapy. ${ }^{15}$

hTERT expression was positively correlated with BM blast \% of ALL patients as well as with CD38 and ZAP 70 among CLL patients. Telomerase activity was positively correlated with the absolute lymphocytic counts among CLL group and negatively correlated with the patient's age of both leukemia groups, otherwise neither hTERT expression nor Telomerase activity shows correlation with any other studied variable. Vural et al, stated that the expression levels of hTERT was higher in ZAP-70 positive CLL patients however this wasn't statistically significant and added that no significant correlation between hTERT with CD38 or ZAP70 was 
reported, only significant correlation with lymphocyte count and age of the patients. ${ }^{21}$ Also, hTERT mRNA was not correlated with hematological parameters or ZAP-70, as reported before. ${ }^{12}$ In concordance to the current study, telomerase activity was correlated significantly with white blood cell and lymphocyte count among but not with bone marrow cellularity or other patient characteristics in CLL patients, as signified by Verstovsek et al. ${ }^{19}$

The limitation of the current work is the small patients' number, as we expect some correlative findings may prove to be significant if applied on larger patients' numbers. We recommend performing larger studies including full prognostic profile of patients that could establish sequential serial detection of hTERT expression and Telomerase activity starting from diagnosis, treatment, remission/ resistance and follow up phases as this would help in identifying the actual role of these parameters in disease progression and prognosis.

One of the insights for future researches, is to evaluate hTERT expression and Telomerase activity in context with chemo-resistance in different groups of acute and chronic lymphocytic leukemia as par example; ALL patients who show poor response to hyper-CVAD, being the most frequently prescribed protocol for ALL adult patients. ${ }^{22}$

As telomerase activity tends to decline obviously during ALL remission phase, consequently it could be further evaluated in larger studies as one of the surrogate parameters for monitoring remission in ALL patients.

As CLL patients with high hTERT expression was reported to have poorer response to usual lines of therapies11. Developing hTERT targeted new therapeutic approaches could ultimately be of special benefit in these patients. Larger studies are still needed to validate the prognostic significance of hTERT expression in different malignancies and open the possibility of the potential success of hTERT targeted therapy. Also the established higher telomerase activity among certain malignancies lightens a rationale for targeting telomerase therapeutically in these diseases.

\section{REFERENCES}

1. O'Sullivan RJ, Karlseder J. Telomeres: Protecting chromosomes against genome instability. Nat Rev Mol Cell Biol 11: 171-181, 2010.

2. Blackburn EH. Telomeres and telomerase: Their mechanisms of action and the effects of altering their functions. FEBS Lett 579: 859-862, 2005.

3. Liu Y, Snow BE, Hande MP, et al. The telomerase reverse transcriptase is limiting and necessary for telomerase function in vivo. Curr Biol 10: 1459-1462, 2000.

4. Kyo S, Takakura M, Fujiwara T, Inoue M. Understanding and exploiting hTERT promoter regulation for diagnosis and treatment of human cancers. Cancer Sci 99: 15281538, 2008.

5. Jones $\mathrm{CH}$, Pepper C, Baird DM. Telomere dysfunction and its role in haematological cancer. $\mathrm{Br} \mathrm{J}$ Haematol 156: 573-587, 2012.

6. Friese CR, Earle C, Magazu LS, et al. Timeliness and quality of diagnostic care for Medicare recipients with chronic lymphocytic leukemia. Cancer 117: 1470-1477, 2011.

7. Franco S, Ozkaynak MF, Sandoval C, et al. Telomere dynamics in childhood leukemia and solid tumors: a followup study. Leukemia 17: 401-410, 2003.

8. Malaska J, Sklenickova M, Krejci K, et al. Telomerase activity and expression and telomere analysis in situ in the course of treatment of childhood leukemias. Blood Cells Mol Dis 26: 534-539, 2000.

9. Tchirkov A, Chaleteix C, Magnac C, et al. hTERT expression and prognosis in B-chronic lymphocytic leukemia. Annals of Oncology 15: 1476-1480, 2004.

10. Ballon G, Trentin L, De Rossi A, Semenzato G. Telomerase activity and clinical progression in chronic lymphoproliferative disorders of B-cell lineage. Leuk Lymphoma 41: 35-45, 2001.

11. Terrin L, Trentin L, Degan M, et al. Telomerase expression in B-cell chronic lymphocytic leukemia predicts survival and delineates subgroups of patients with the same $\operatorname{lgVH}$ mutation status and different outcome. Leukemia 21: 965-972, 2007.

12. Abdelrahman Z, Abou shamaa L, Sultan M, et al. Expression of CLL upregulated gene1(CLLU), telomere reverse transcriptase (hTERT), zeta-associated protein kinase -70 (ZAP-70) in patients with chronic lymphocytic leukemia. J Immunol 196: (1 Supplement) 144.10, 2016.

13. Ulaner GA, Hu JF, Vu TH, et al. Tissuespecific alternate splicing of human telomerase reverse transcriptase (hTERT) influences telomere lengths during human development. Int J Cancer 91: 644-649, 2001.

14. Kleideiter E, Bangerter U, Schwab M, et al. Telomeres and telomerase in paediatric patients with T-cell acute lymphoblastic leukaemia (T-ALL). Leukemia 19: 296-298, 2005. 
15. Nowak T, Januszkiewicz D, Zawada M, et al. Amplification of hTERT and hTERC genes in leukemic cells with high expression and activity of telomerase. Oncol Rep 16: 301-305, 301, 2006.

16. Kustanovich A, Savitskaya T, Potapnev M. Telomerase activity and telomere length in malignant cells of children with acute lymphoblastic leukemia. Experimental oncology 25: 69-73, 2003.

17. Arora B, Kumar L, Das BC, et al. Telomerase activity and human telomerase reverse transcriptase mRNA expression in acute lymphoblastic leukemia. J Clin Oncol 22: 14 Suppl, 6705-6705, 2004

18. Trentin L, Ballon G, Ometto L, et al. Telomerase activity in chronic lymphoproliferative disorders of $\mathrm{B}$-cell lineage. $\mathrm{Br}$ J Haematol 106: 662-668, 1999.

19. Bruedigam C, Lane SW. Telomerase in hematologic malignancies. Curr Opin Hematol 23: 346-353, 2016.

20. Rampazzo E, Bonaldi L, Trentin L, et al. Telomere length and telomerase levels delineate subgroups of B-cell chronic lymphocytic leukemia with different biological characteristics and clinical outcomes. Haematologica 97: 56-63, 2012.
21. Vural F, Karaca E, Soyer N, et al. Comparison of CD38, ZAP70 and hTERT expression with known prognostic markers in patients with chronic lymphocytic leukemia during five-year follow-up period. UHOD 24: 179-184, 2014.

22. Buyukasik $\mathrm{Y}$, Acar $\mathrm{K}$, Kelkitli E, et al. Hyper-CVAD regimen in routine management of adult acute lymphoblastic leukemia: a retrospective multicenter study. Acta Haematol 130: 199-205, 2013.

\section{Correspondence:}

Dalia Gamil AMIN, M.D.

Cairo University, Faculty of Medicine

Department of Clinical Pathology

CAIRO / EGYPT

Tel: 0201221692385

02-3635554

e-mail: aeagipy@yahoo.com 